\title{
ESTRATEGIAS DIDÁCTICAS EN TECNOLOGÍAS WEB PARA AMBIENTES VIRTUALES DE APRENDIZAJE
}

\author{
TEACHING STRATEGIES IN WEB TECHNOLOGIES FOR VIRTUAL LEARNING \\ ENVIRONMENTS
}

\author{
Ilber Dario Saza-Garzón 1
}

\begin{abstract}
RESUMEN
Los Ambientes Virtuales de Aprendizaje (AVA) han sido un tema de discusión y de interrogantes sobre todo en la búsqueda de las mejores prácticas docentes, de cuáles herramientas puede usar y cómo darles el óptimo aprovechamiento para lograr tener mejores resultados en la educación virtual, por lo tanto en este documento se exponen algunos elementos sobre las características, antecedentes, didácticas, estudios que tiene los ambientes virtuales y aplicaciones de la web como herramientas de apoyo a los procesos de enseñanza y aprendizaje, para que un tutor virtual tenga en cuenta a la hora de planificar, diseñar, crear e implementar cursos virtuales. En consecuencia, el lector encontrará conceptos, explicaciones y diferentes procesos evolutivos que ha sufrido las TIC y como estas se han involucrado en el contexto educativo, divisando sus posibles aplicaciones desde la mediación de la didáctica, además se exponen algunas sugerencias de cómo llevar a cabo el uso de las mismas en los ambientes virtuales de aprendizaje para fortalecer los diferentes procesos de enseñanza y aprendizaje.
\end{abstract}

Palabras clave: Ambientes virtuales de aprendizaje, educación virtual, Entornos virtuales de aprendizaje, Estrategias didácticas en ambientes virtuales de aprendizaje, e-learning, Tecnología educativa.

\begin{abstract}
The virtual learning environments (AVAs) have been a subject of discussion and questions mainly on finding the best teaching practices, which tools you can use them and how to achieve optimum utilization have better results in virtual education, for Therefore in this paper some elements about the characteristics, history, teaching, studies have virtual environments and web applications as tools to support teaching and learning, are set for a virtual tutor note the when planning, designing, creating and implementing online courses. Thus the reader will find concepts, explanations and different evolutionary processes that wins ICT and how are you have been involved in the educational context, spotting potential applications from mediation of teaching, plus some suggestions of how to carry out exposed use thereof in virtual learning environments to strengthen the different processes of teaching and learning.
\end{abstract}

Keywords: Virtual learning environments, virtual education, teaching strategies in virtual learning environments, e-learning, educational technology.

Fecha de recepción: Febrero 04 de 2016 / Fecha de aceptación: Mayo 17 de 2016

Tipología: Artículo de Reflexión

Para citar este artículo: Saza, G. I. (2016). Estrategias didácticas en tecnologías web para ambienes virtuales de aprendizaje. Praxis. Vol. 12, 103-110

1. Magister en Tecnologías de la Información aplicadas a la Educación. Docente UNIMINUTO, Bogotá-Colombia. Correo: isaza@uniminuto.edu, dariosaza82@ gmail.com 


\section{INTRODUCCIÓN}

$\mathrm{E}$ presente documento hace un análisis de los aspectos didácticos que debe tener un ambiente virtual de aprendizaje (AVA), en donde se evidencian algunos elementos didácticos que puede orientar a un tutor virtual para la planeación de las aulas virtuales. Por lo tanto, este documento parte haciendo algunas aclaraciones sobre didáctica y didáctica general para ambientes virtuales de aprendizaje.

Posteriormente hace un breve recorrido sobre el proceso evolutivo de la educación virtual y de los ambientes virtuales de aprendizaje, en donde se toma aspectos relevantes del origen de los mismos desde la educación a distancia, evidenciando las características que tiene hoy en día la educación virtual comparadas con la educación presencial.

Con lo anterior, se parte de los aportes de algunos autores de las necesidades que tiene la educación virtual desde la planeación, organización e implementación, hasta las diferentes estrategias didácticas mediadas por las TIC que puede usar un docente para aplica dentro de los AVA, por lo tanto, este documento hace un análisis de los aspectos didácticos que se deben tener en cuenta para optimizar el uso de las aulas virtuales y fortalecer los procesos de enseñanza y aprendizaje.

\section{METODOLOGÍA}

Hablar de ambientes virtuales de aprendizaje y cómo debe ser un curso virtual, desde lo metodológico, requiere hacer la reflexión pedagógica sobre cuáles y qué metodos deben darse en una clase desde lo virtual, en donde los aspectos como distancia, comunicación y espacio físico cambian totalmente en estos entornos. Por lo tanto, es necesario repensar si las practicas docentes que se hacen en lo presencial se pueden aplicar en lo virtual y conocer si tiene el mismo resultado o si se requiere de nuevas formas de dar clase y por ende nuevos perfiles de docentes. Algunas aproximaciones a esos nuevos perfiles docentes las exponen Cardona y Vaca González (2014), los cuales dicen que "Hoy en día se requiere de docentes con nuevas competencias y habilidades digitales en pro de la generación de aprendizajes y procesos comunicativos eficaces y eficientes” (p. 50).

Es por ello que surgen los interrogantes de ¿cómo se puede llevar un curso virtual desde lo didáctico y tecnológico para estudiantes de maestría?, ¿qué requerimientos didácticos y pedagógicos son necesarios para los ambientes virtuales de aprendizaje? En ese orden de ideas, la metodología de investigación que se trabajó en el proyecto titulado: PROPUESTA DIDÁCTICA PARA LA MODALIDAD E-LEARNING DEL PROGRAMA MAESTRÍA EN TECNOLOGÍAS DE LA INFORMACIÓN APLICADAS A LA EDUCACIÓN, EN EL CASO TALLER ESPECIFICO II, implementado en la Universidad Pedagógica Nacional (UPN) fue de carácter cualitativo de alcance descriptivo con un diseño metodológico basado en los postulados de estudio de caso de Robert Stake, cuyo objeto de estudio era postular una propuesta didáctica desde el constructivismo endógeno y exógeno para un ambiente virtual de aprendizaje, en una asignatura que corresponde a la Maestría en tecnologías de la información aplicadas a la educación de la Universidad Pedagógica Nacional.

\section{RESULTADOS}

La investigación se llevó a cabo en los periodos académicos 2013-2, 2014-1 y 2014-2, y la toma de datos fueron por medio del instrumento de protocolos verbales, los resultados arrojados se centraron en el modelo didáctico, uso de las herramientas TIC y como estas se relacionan en la propuesta didáctica desde lo pragmático en el AVA, por lo tanto, los resultados arrojados fueron:

El Modelo didáctico con sus respectivas estrategias didácticas y herramientas tecnológicas, tuvieron un comportamiento favorable, porque se evidenció un uso adecuado de los diferentes elementos, los organizadores previos cumplieron con el propósito de ilustrar a los estudiantes en los temas más generales que proponen el aula, además la participación de los distintos foros permitió la reflexión y acercamiento a los conceptos 
que ya poseen y poderlos relacionar con lo nuevos. Esta estrategia ha sido estudiada por diversos autores, como es el caso de Ausubel y Mayer (como los citó Peñalosa, 2013) Afirma que:

Una estrategia general para iniciar la cobertura de una unidad o un tema de un curos es la presentación o activación de los conocimientos previos, la intención de esto es que los estudiantes relacionen los contenidos que se presentan con lo que ya saben. (p. 125)

La activación cognitiva esta mediada por una WebQuest, esta estrategia didáctica orienta y explica las actividades que debe realizar el estudiante, este recursos orientó de forma adecuada a los participantes puesto que se muestra de un forma estructurada y que a su vez el estudiante encuentra toda la información concentrada en una solo sitio. Según Bautista, Borges, \& Forés (2006) exponen:

Una WebQuest se presenta en una página web que tiene una estructura bien definida y que no suele ser complicada, aunque la cantidad de información y de enlaces a los que se puede acceder a partir de ella depende de su creador. (p. 147)

Por otra parte, se evidencia la necesidad de acompañamiento y constante comunicación del docente frente a los estudiantes que se les dificulta más el aprendizaje, lo anterior surge de las preguntas que suscitaron del foro, es por ello que el docente tutor debe estar en constante comunicación y acompañamiento en el proceso de aprendizaje de los integrantes de aula para responder a las dudas que suscitan en el proceso.

Agregando a lo anterior, se evidencia que las TIC como herramienta de apoyo a los procesos de aprendizaje permite también crear nuevos espacios y nuevas formas de presentar la información y de distribuir, exponer y clarificar los contenidos plasmados, así mismo de la realización y entrega de actividades. Según López (2013):

La presencia de las TIC como apoyo a la docencia hace posible traspasar los muros escolares y llegar a lugares donde se encuentran aquellos que más lo necesitan. Es evidente que estas tecnologías dan lugar a un cambio de paradigma que se refleja, de manera evidente en el cambio curricular de muchas instituciones. (p. 234)

Es por ello que las TIC permitieron a los estudiantes tener un fácil acceso a la información en donde se presentó de múltiples formas, teniendo en cuanta la estrategia didáctica y las necesidades de los estudiantes. Sin embargo, es necesario conocer las funciones y posibles aplicaciones que se pueden dar en el contexto educativo, puesto que estas por si solas sin la intención pedagógica no cumplen con el propósito de herramienta de apoyo a los procesos de enseñanza y aprendizaje.

\section{DISCUSIÓN}

Hablar del método o mejor aún de didáctica requiere hacer algunas aproximaciones conceptuales que son pertinentes para hablar de una didáctica específica en ambientes virtuales de aprendizaje. De ahí que, la didáctica desde la etimología proviene del verbo griego didásko, que significa enseñar, algunos autores hablan del método y del cómo enseñar, por ejemplo. Comenio, en el libro Didactica Magna la define como el artificio universal para enseñar, sin embargo la didáctica ha sido objeto de estudio de muchas disciplinas y ciencias y ha sido aplicada en distintas áreas.

La didáctica se construye desde los protagonistas del acto educativo (educador y estudiante) y por medio de las interacciones comunicativas se enriquece. Por lo tanto la didáctica es entendida como la disciplina pedagógica aplicada, es decir la practica de las teorías pedagógicas. Según Medina \& Salvador (2009) "La didáctica es la disciplina o tratado riguroso de estudio y fundamentación de la actividad de enseñanza en cuanto propicia el aprendizaje formativo de los estudiantes en los más diversos contextos” (p.7). en consecuencia la didáctica busca el constante mejoramiento de los procesos de enseñanza y aprendizaje. 
Por otra parte, es necesario tener claro que una parte esencial de la didáctica es el método (¿cómo enseñar-aprender?) y este se vale de estrategias que pueden ser para el docente, estudiante, contenido y contexto, en ese orden de ideas, el proceso didáctico se centra en responder la pregunta de: ¿Cómo enseñar o cómo actuar? Y para responder esta pregunta existen diversos caminos o métodos, estos consisten en una serie de decisiones y acciones ejecutadas por el docente, con el fin de tener elementos de cómo enseñar y como aprender, los métodos se basan en estrategias tanto para la enseñanza (docente) como de aprendizaje (estudiante) y que a su vez contiene técnicas. Según Medina \& Salvador (2009): "el método se concreta en una variedad de modos, formas, procedimientos, estrategias, técnicas actividades, y tareas (de enseñanza y aprendizaje). En funciones de estas variaciones se establecen diversos tipos de métodos" (p. 172).

Teniendo en cuenta lo anterior, se puede entender la didáctica como arte o técnica, ya que esta nos da parámetros claros de cómo llevar a cabo una clase, pero también la didáctica es ciencia porque no se puede separar de la teoría, además que permite abrir nuevos espacios para la investigación, en donde diversos saberes han identificado nuevos fenómenos para ser estudiados y por consiguiente surgen nuevas formas y estrategias de cómo mejorar los procesos de enseñanza y aprendizaje. Díaz (2002) dice que "la didáctica es un saber científico porque: Recibe aportaciones de otras ciencias. Intenta elaborar teorías descriptivas (...) la didáctica es un saber técnico porque: se nutre de las normas, leyes o reglas derivadas del saber científico y del tecnológico” (p. 37). En ese orden de ideas se puede definir didáctica como una disciplina orientada a la práctica, por lo que requiere de estrategias de enseñanza, aprendizaje y de formación.

Agregando a lo anterior, el objeto de la didáctica son los procesos de enseñan y el aprendizaje del que se valen mediante diversas estrategias, tanto para el docente como para el estudiantes; además, la didáctica puede ser a nivel general o nivel particular. La didáctica general se aplica a todo acto educativo en donde abarca los principios, métodos, procedimientos y técnicas aplicables a eventos reales, y la acción de la didáctica parte de planteamiento, ejecución y verificación.

Ahora bien, antes de precisar las diferencias entre ambientes virtuales de aprendizaje y ambientes tradicionales (salón de clase físico) es necesario hacer aproximaciones de su origen, como es bien sabido, las Tecnologías de la información y comunicación (TIC) han cambiado sustancialmente nuestro estilo de vida en menos de 20 años, hemos sufrido cambios en diferentes contextos como laboral, cultural y educativo, este ultimo las TIC han evolucionado e ingresado en las instituciones educativas, en sus comienzos en la enseñanza de computación en los años 90, pasando por la conectividad a la WWW y el uso del hipervínculo, hasta apoyo para los diferentes procesos de aprendizaje de las diferentes áreas de conocimiento gracias a los diferentes desarrollos de la WEB 2.0 y 3.0, pero la educación virtual tiene sus inicios de la educación a distancia y los procesos evolutivos de las TIC, estas han creado nuevos medios y formas de enseñar y aprender, estas formas son conocidos como: E-learning (aprendizaje electrónico), b-learning (aprendizaje semi-presencial o combinado), m-learning (aprendizaje desde celulares o móviles), Networklearning (aprendizaje en la red).

Ahora bien, los nuevos medios para enseñar y aprender requieren de plataformas o lugares en la Web para planificar, diseñar e implementar un curso, estos son llamados ambientes virtuales de aprendizaje (AVA) o Entornos virtuales de enseñanza y aprendizaje (EVEA) y estos ambientes requieren de elementos tecnológicos como plataformas, pero también de elementos didácticos. A continuación, se realizará una breve comparación entre las características de los AVA y los cursos o ambientes tradicionales.

Los ambientes virtuales de aprendizaje tienen elementos que los hacen totalmente diferentes a entornos académicos tradicionales, algunos de estos son el espacio físico; en un ambiente virtual no existe como tal, en consecuencia el espacio es una plataforma de sistema de gestión de aprendizaje (LMS) entre los cuales hay plataformas como Moodle, blackboard, chamilos entre otros, 
los cuales funcionan como un medio para alojar archivos, actividades y comunicación, además presta servicios de alojamiento y repositorios de tareas, prestando herramientas de retroalimentación y de comunicación para compartir conocimiento, esta plataforma requiere que los estudiantes y docentes estén en constante conexión. Según Cukierman, Rozenhauz \& Santángelo (2009) "dicho elemento funcional será el que permitirá la administración y gestión de las actividades de enseñanza y-aprendizaje” (p.53).

Otro elemento que diferencia los ambientes virtuales de aprendizaje de los presenciales es la flexibilidad en los horarios, debido a que este se ajusta a los tiempos disponibles de cada uno de los participantes, cada integrante del aula elige a que momento puede ingresar al aula a participar, leer, realizar y compartir las actividades que allí se plasman, es importante recalcar que la autonomía y responsabilidad del estudiante juegan un papel importante para asegurar el éxito del proceso formativo.

Además, los LMS resuelven la problemática de la distancia, puesto que en un ambiente virtual de aprendizaje puede haber estudiantes de diferentes partes del mundo, haciendo que las interacciones surgan desde la multiculturalidad, en algunas ocasiones se puede persibir las distancia como sinónimo de abandono, pero no es así, la labor docente es de acompañamiento constante en las diferentes situaciones de dificultad que presenten los participantes. Según Barberá (2008) “El tutor desarrolla este proceso de seguimiento continuado ofreciendo los apoyos y soportes que se requieran en momentos muy determinados” (p.49). Sin embargo, es el estudiante el único responsable de los procesos de aprendizaje, es por ello que el ingreso al aula debe ser constante y no esporádico.

Por ultimo, la comunicación en ambientes virtuales de aprendizaje cobra gran relevancia, ya que esta es asincrónica, y se evidencia en los procesos de exposición de temas y contenidos, porque no todos se conectan al mismo tiempo para recibir la información; en cuanto a la comunicación entre estudiantes y docente depende de las disposiciones y necesidades de cada participante, debido a ello la comunicación debe ser multidireccional, esta forma permite que cada estudiante participe de forma autónoma, debido a que cada participante tiene necesidades e inquietudes particulares. Según Bautista, Borges, \& Forés (2006) "la comunicación en red o multidireccional es la que realmente demuestra una participación activa y autónoma del estudiante” (p.129).

Lo anterior permite también crear espacios de socialización de vivencias, saberes y conocimientos, en donde el docente debe hacer un ejercicio de planear estos espacios de comunicación y socialización para la construcción de conocimiento, generación de ejercicios argumentativos por medio de la discusión y debate que enriquezca los saberes aprendidos. Ureña y Villalobos (2011). Exponen

La socialización del conocimiento en los institutos universitarios consiste en la relación comunicativa que se establece entre dos o más participantes y facilitadores a través de la conversación donde se comparten experiencias, valores, emociones, principios o sentimientos en torno a la situación o tema objeto de la reunión. (p. 161)

Sin embargo, existen también obstáculos o problemáticas en los ambientes virtuales de aprendizaje entre los cuales están:

- Brecha Digital: Muchos de los docentes aún se niegan a acercasen a las TIC, no conocen el funcionamiento de un computador y por ende se les dificulta utilizar las diferentes aplicaciones Web para poder facilitar los procesos de enseñanza aprendizaje de determinado saber. Prefieren seguir con las mismas prácticas docentes de hace 20, 30 y 40 años. En ese orden de ideas los intereses de los estudiantes van por un camino y los saberes del docente van por otro.

- Ética y valores: Debido a que en los ambientes virtuales de aprendizaje no se hacen clases magistrales "tradicionales" si no que requiere que sus participantes sean autónomos, responsables en el estudio de los diferentes contenidos y requiere que el aprendizaje sea una construcción colectiva partiendo del aprendizaje individualizado, en donde cada 
estudiante debe ser transparente y responsable de su proceso de aprendizaje; transparente en el sentido de presentar trabajos, actividades y evaluaciones desde el estudio y comprensión de los temas y no desde la copia de archivos y ayuda de terceros en la presentación de evaluaciones.

- Calidad: Son muchos las críticas de la educación virtual, generando interrogantes si un estudiante aprende lo necesario desde lo virtual como lo hace un estudiante en lo presencial, se hacen criticas fuertes a las evaluaciones, por lo tanto se debe garantizar que los ambientes virtuales de aprendizaje deben estar bien organizados, planeados, con evaluaciones claras y con determinada cantidad máxima de estudiantes, con el fin que el docente haga las respectivas revisiones y retroalimentación a los procesos y progresos de cada estudiante, por lo tanto el tiempo que el docente requiere para este seguimiento debe ser mayor al destinado en una clase presencial. La educación virtual no se trata de llenar de estudiantes un curso, se trata de estimar los tiempos requeridos para poder hacer un seguimiento serio a los procesos de aprendizaje de cada integrante, con el fin de garantizar la calidad educativa.
En consecuencia a lo anterior, se debe partir desde la capacitación docente el cual debe tener conocimientos en herramientas tecnológicas proporcionadas por la web y como estas sirven para apoyar los procesos de enseñanza y aprendizaje, estas permiten compartir, exponer, explicar comunicar orientar simular y evaluar entre otros procesos de enseñanza y aprendizaje, pero estas no son suficientes si no se tiene en cuenta el componente pedagógico, en otras palabras las herramientas tecnológicas por si solas no enseñan, debe tener la intención pedagógica y didáctica para que estas apoyen los diferentes procesos de enseñanza y aprendizaje.

Las herramientas tecnológicas de la web que permiten al usuario crear, subir, compartir y editar información. Algunos autores como Nafria (2008) hace un descripción de herramientas WEB 2.0 mostrando algunas aplicaciones en determinados contextos o necesidades, otros como Revuelta \& Pérez (2011) categorizaron las de herramientas web 2.0 según sus utilidades y funciones. La siguiente tabla es el resultado de la categorización y uso de herramientas usada en la experiencia, en donde muestra algunas herramientas Web 2,0, en ella aparece las categorías y nombre de las herramientas Web.

Tabla 1.

Categorización de Herramientas Web

\begin{tabular}{|c|c|}
\hline Categoría & Herramientas Web \\
\hline Presentaciones & $\begin{array}{c}\text { SlideShare } \\
\text { Calameo } \\
\text { Issuu }\end{array}$ \\
\hline $\begin{array}{c}\text { Esquemas, diagramas o instrumentos de } \\
\text { conocimiento }\end{array}$ & $\begin{array}{c}\text { Cacoo } \\
\text { Cmaptools } \\
\text { Lucidchart }\end{array}$ \\
\hline Disco / Almacenamiento & $\begin{array}{c}\text { Dropbox } \\
\text { Google DRIVE }\end{array}$ \\
\hline Líneas de Tiempo & Timerime \\
My Timeline \\
\hline Documentos / Office & Google DRIVE \\
& SKYDRIVE \\
\hline
\end{tabular}


Continuación Tabla 1.

\begin{tabular}{|c|c|}
\hline Categoría & Herramientas Web \\
\hline Video / animaciones & YouTube \\
& Vimeo \\
& Animoto \\
\hline & Hangouts \\
Comunicación & Skype \\
& Foros \\
\hline
\end{tabular}

Fuente: Elaboración propia basada en Pérez (2011)

Estas categorías son una orientación para el docente a la hora de seleccionar herramientas de la Web como herramienta de apoyo a los procesos de enseñanza y aprendizaje que surgen en los diferentes contextos académicos, sin embargo la anterior lista es una muestra de las múltiples herramientas que puede encontrar en la Web.

Otros autores ubicaron las tecnologías web según el propósito pedagógico y empezaron hablar de estrategias didácticas apoyadas por TIC, en este caso Díaz \& Hernández (2010) hablan de estrategias de enseñanza en el marco del modelo constructivista y estas a su vez hacen clasificaciones según la intencionalidad, algunas de ellas son:

- Estrategias para activar conocimientos previos, los cuales se pueden utilizar material multimedial o elementos organizativos como mapas conceptuales.

- Estrategias para Organizar la información, las tecnologías que se pueden aplicar en este tipo de estrategias se encuentra en los esquemas, como son mapas conceptuales, mentefactos, cuadros sinópticos, diagramas de flujo, lineas de tiempo, entre otros

- Estrategias para promover la enseñanza situada, entre las cuales situamos aprendizaje basado en problemas en donde se puede exponer un caso expuesto en herramientas como son presentaciones, materiales multimediales (videos, imágenes, audios). También se situá aprendizaje mediante proyectos en los cuales se pueden crear revistas, wikis, entre otros.
Por otra parte, autores como Peñalosa (2013) afirman que:

el modelo de aprendizaje auténtico contextualizado puede servir de base para el diseño de cursos (...) el modelo contempla el diseño de actividades relacionadas con las cuatro familias de estrategias, en cada una de las cuales se recomienda el uso de tecnologías digitales. (p. 103).

Estas estrategias son: Establecimiento de contexto que consiste en situar al estudiante en un contexto real, entre las herramientas más usadas son los videos e ilustraciones. La segunda estrategia es la presentación de conocimiento, esta consiste en hacer explicaciones o demostraciones del tema a tratar, entre los cuales están las presentaciones y diagramas. La tercera es de aplicación y construcción de conocimiento, que cosiste en que los estudiantes realicen determinadas actividades para reforzar lo explicado, las herramientas más usadas son las de creación de mapas conceptuales, presentaciones y lecturas. Por último la estrategia de reflexión e integración de conocimiento, se trata de la reflexión dada del proceso anterior y exponen lo aprendido para hacer la respectiva retroalimentación, las herramientas más usadas son los foros y debates.

Teniendo en cuenta lo anterior, es necesario que el docente tenga competencias en los aspectos pedagógicos y tecnológicos, por lo tanto el docente al momento de diseñar y crear un ambiente virtual de aprendizaje debe tener en 
cuenta la planeación del aulas desde lo tecnológico, metodológico, pedagógico y evaluativo, el cual implica organizar todos los elementos que la conforman tratando de dar una estructura lógica que le permita al estudiante tener una navegabilidad óptima permitiendo así cumplir con los objetivos trazados. Bracho y Carruyo. (2011) definen

la planificación implica y aspira implementar orden y dirección en los procesos conforme a las pautas establecidas, de allí que tiene una doble connotación; una lógica de donde se deriva el conocer y la otra operativa, que indica la forma de actuar para lograr el objetivo deseado (p. 21)

Ahora bien, para llevar la planeación de AVA es necesario contar con un diseño Instruccional, este formato permite planificar, organizar desde lo curricular, tecnológico y pedagógico un curso virtual, se tienen en cuenta las teorías y metas de aprendizaje, basados en actividades y especificando los tiempos y recursos para cumplir con los objetivos propuestos. Según Santoyo (2005) dice que "el diseño Instruccional tiene por objetivo el análisis de: las condiciones de aprendizaje, los aprendices, el contexto de aprendizaje y las tareas, las especificaciones de los objetivos de aprendizaje y, finalmente, la evaluación” (p.87).

En conclusión, las plataformas tecnológicas y herramientas web por si solas no suplen la labor y mediación que debe hacer el docente, es por ello que un aula virtual puede estar bien diseñada desde lo tecnológico pero si desde el saber pedagógico y didáctico para crear una buena propuesta no sirve de nada, las tecnologías no son el fin son el medio para cumplir con las metas de aprendizaje, otro aspecto de vital importancia en el compromiso de todos los participantes del aula virtual (docentes-estudiantes) para lograr los objetivos propuestos, en ese orden de ideas se requiere de un docente reflexivo frente a su que hacer educativo competente en el saber pedagógico y tecnológico y un estudiante responsable, ético y critico frente a sus procesos de aprendizaje, capaz de interrogar, compartir y construir conocimiento.

\section{REFERENCIAS BIBLIOGRAFICAS}

Barberá, E. (2008). Aprender e-learning. Barcelona: Paidós.

Bautista, G., Borges, F., \& Forés, A. (2006). Didáctica universitaria en entornos virtuales de enseñanza-aprendizaje. Madrid: Narcea.

Bracho Pérez, K., \& Carruyo Duran, N. (2011). Planificación estratégica y gestión del gerente educativo en instituciones de educación primaria. Praxis, 7(1), 13-29. doi:http://dx.doi. org/10.21676/23897856.5

Cardona Bedoya, J., \& Vaca González, Y. (2014). Estrategias de la unidad de negocio prestar servicios de formación virtual - unipanamericana compensar. Praxis, 10(1), 48-59. doi:http:// dx.doi.org/10.21676/23897856.1358

Cukierman, U. Rozenhauz, J. \& Santángelo, H. (2009). Tecnología educativa, recursos, Modelos y Metodologías. Buenos Aires. Editorial Pearson.

Díaz, F (2002). Didáctica y currículo: un enfoque constructivista. Univ de Castilla La Mancha.

Díaz, F., \& Hernández, G. (2010). Estrategias docentes para un aprendizaje significativo: una interpretación constructivista. México, D.F.: McGraw Hill.

López, M. (2013). Aprendizaje, competencias y TIC: aprendizaje basado en competencias. México, D.F.: Pearson.

Medina, A., \& Salvador, F. (2009). Didáctica general. Madrid. Pearson Prentice Hall : UNED

Nafria, I. (2008). Web 2.0: El usuario, el nuevo rey de Internet. Barcelona. Gestión 2000.

Peñalosa Castro, E. (2013). Estrategias docentes con tecnologías: guía práctica. Naucalpan de Juárez, México: Pearson.

Revuelta, F. Domínguez, \& Lourdes Pérez, L. (2011). Interactividad de los entornos en la formación on-line. Editorial UOC.

Santoyo, C. (2005). Alternativas Docentes Volumen III. Análisis y evaluación de habilidades metodológicas, conceptuales y profesionales en la formación del psicólogo. México D. F. Universidad autónoma de México.

Ureña Villamizar, Y., \& Villalobos de Weffer, R. (2011). Gestión del conocimiento en institutos universitarios de tecnología. Praxis, 7(1), 155-180. doi:http://dx.doi.org/10.21676/23897856.20 